\title{
An Extended Maxwell Fluid Model in Terms of Dimensionless Relaxation Time in Polymeric Non-Newtonian Liquids Motion
}

\author{
K. Boubaker \\ Department of Physics \& Chemistry, School of Sciences and Technology of Tunis (ESSTT), University of Tunis, Tunisia
}

\begin{abstract}
Due to the inherent nonlinear nature of viscoelastic non-Newtonian fluids, one of the challenging aspects for developing and utilizing their related devices to achieve high performance is the development of models that can accurately describe their particular characteristics. In the past three decades, the models for viscoelastic fluids have been focused on how to improve the modeling accuracy and reliability. In this study, the classical Maxwell model for viscoelastic fluids is extended in terms of characteristic relaxation time strain-dependence. The extended model is very useful in describing the response of geological and many other polymeric fluids. A self-consistent extended Maxwell model for viscoelastic non-Newtonian fluids is presented. By way of example we consider the exact solution of the extended Maxwell model, describing the standard dashpot-spring configuration.
\end{abstract}

Keywords Viscoelasticity, Maxwell Fluid, Non-Linearly Elastic Body, Non-Linear Dashpot, Extended Implicit Model

\section{Introduction}

The classical Maxwell fluid model, known as a viscoelastic rate type model, has been introduced by Maxwell[1] in his investigations on the dynamical theory for gases. The earliest fundaments to this model referred to the manner a body stores energy and produces entropy. Later, a simple mechanical analog[2,4], implemented the standard formulation to the one dimensional (Fig. 1)..

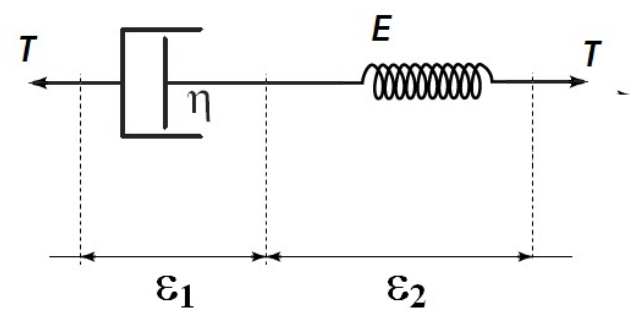

Figure 1. Mechanical analog for the actual model

Rao et al. [5], showed that this model was appropriated for describing polymeric liquids motion. This model considers that both elastic and viscous stress depend on strain. Nevertheless, its use was restricted to dilute

* Corresponding author:

mmbb11112000@yahoo.fr (K. Boubaker)

Published online at http://journal.sapub.org/fs

Copyright (C) 2012 Scientific \& Academic Publishing. All Rights Reserved polymeric fluids where with relatively short relaxation time. In this paper, and oppositely to the presumptions of Maxwell[1], fluid viscosity and the relaxation time depend on the invariants associated with the stress along with Cauchy-Green stretch tensor or tensors from the appropriate natural configurations. In fact, the thorough study of some polymer melts planar Couette and Poiseuille flows[6], showed that such fluids effective relaxation time depends on the stress and hence may vary in wide ranges.

\section{Mathematical Formulation of the Problem}

According to the model[7-9], the components of the constituents, namely an energy storing mechanism that is modelled in one dimension as a spring, and the dissipative constituent that is modeled in one dimension as a dashpot, which is assumed to be in series with the spring (Fig. 1). The fact that the spring and dashpot are in series leads to the trivial assumptions, hence, in the classical Maxwell one dimensional constitutive model, and in concordance with the notation of Figure 1, the total strain $\varepsilon$ is given by:

$$
\varepsilon=\varepsilon_{1}+\varepsilon_{2}
$$

where $\varepsilon_{1}$ and $\varepsilon_{2}$ are the elongations, in the dashpot and spring respectively.

The stress in the dashpot and spring, respectively $T_{1}$ and $T_{2}$, verify the disposition-linked relation: 


$$
T_{1}=T_{2}=T
$$

From the constitutive equation for the dashpot and the spring:

$$
\left\{\begin{array}{l}
T_{1}=\eta \dot{\varepsilon}_{1} \\
T_{2}=E \varepsilon_{2}
\end{array}\right.
$$

By combining Eq. (1-3), we obtain Maxwell Equation:

$$
T+\frac{\eta}{E} \dot{T}=T+\xi \dot{T}=\eta \dot{\varepsilon}
$$

where $\xi$ denotes, in the earliest model, a material constant that has the units of time, or the so-called "relaxation time".

Maxwell original model is based on the idea of linear viscoelasticity, which assumes that for small strains, the relaxation modulus is independent of the strain but only depends on the elapsed time. For quantifying this dependence, Macosko[10] defined the relaxation modulus $\Gamma(t, \varepsilon)$ as the ratio between the stress and the strain:

$$
\Gamma(t, \varepsilon)=\frac{T(t, \varepsilon)}{\varepsilon}
$$

An efficient way to quantify the phenomenon of viscoelasticity consists of evaluating the limit values of the relaxation modulus, the instantaneous elastic modulus:

$$
\Gamma_{0}=\left.\frac{T(t, \varepsilon)}{\varepsilon}\right|_{t=0}
$$

and the asymptotic elastic modulus:

$$
\Gamma_{\infty}=\left.\frac{T(t, \varepsilon)}{\varepsilon}\right|_{t \rightarrow \infty}
$$

For viscoelastic materials, it has been recorded that stress decreases exponentially[7-9].

\section{Results and Discussion}

\subsection{The Extended Maxwell Fluid Model}

According to the results recorded by Larson et al.[6], Gorodtsov et al.[7], M. Graham[8] and Keiller[9] , $\xi$ denotes, in the earliest model, a material constant that has the units of time. In the actual model, the relaxation time is considered as a strain-dependent variable. This configuration has been evoked earlier by Pipkin et al.[11] through the single step strain history model, express by:

$$
\left\{\begin{array}{l}
\hat{\xi}(\varepsilon)=\phi_{0}+\phi_{1} C+\phi_{2} C^{2} \\
C=F^{T} F
\end{array}\right.
$$

where $\hat{\xi}(\varepsilon)$ denotes the relaxation time estimator $\phi_{0}, \phi_{1}$ and $\phi_{2}$ are constants, $C$ is the strain dependent tensorial relaxation function and $F$ is the deformation gradient tensor.

In the actual model, a polynomial scheme is proposed for modeling both relaxation time $\xi(\varepsilon)$ and stress $T(t, \varepsilon)$ in respect to the inherent boundary conditions imposed by Eq. 8.

\subsection{The Boubaker Polynomials Expansion Scheme}

The main equation of the model is:

$$
T+\xi(\varepsilon) \frac{\partial T}{\partial t}=\eta \frac{\partial \varepsilon}{\partial t}
$$

At this level, The Boubaker Polynomials Expansion Scheme BPES[12-21] is applied through setting the expression:

$$
\xi(\varepsilon)=\frac{1}{2 N_{0}} \sum_{k=1}^{N_{0}} \lambda_{k} \times B_{4 k}\left(r_{k} \varepsilon\right)
$$

where $B_{4 k}$ are the $4 k$-order Boubaker polynomials, $r_{k}$ are $B_{4 k}$ minimal positive roots, $N_{0}$ is a prefixed integer, $\left.\lambda_{k}\right|_{k=1 . . N_{0}}$ are unknown pondering real coefficients. Figure 2 presents relaxation time strain-dependent variations for both actual and single step strain history[11] models.

u. a.

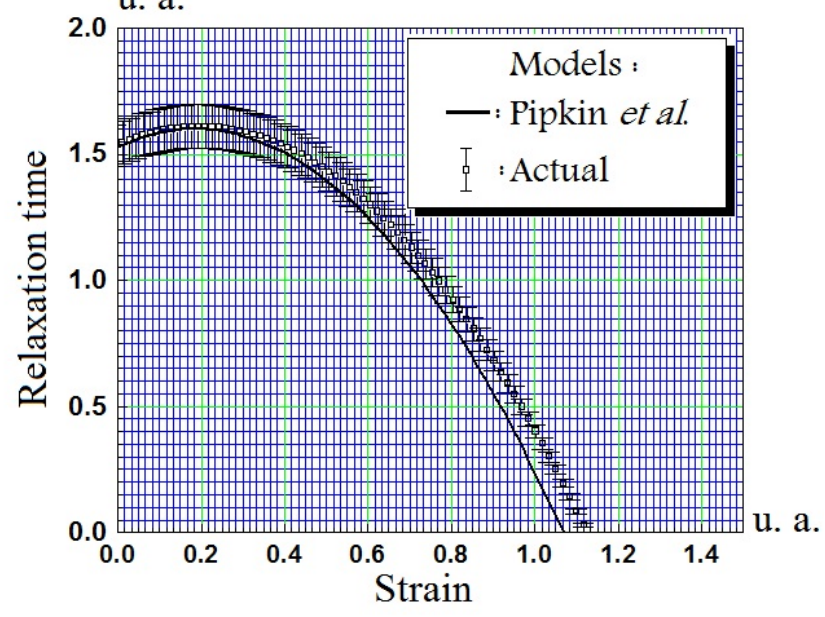

Figure 2. Strain-dependent relaxation time for the two related models

The proposed model is tested tough stress responses to a Heaviside step function $H(0)$ excitation. Consequently, it comes for Eq. (9) that:

$$
\left\{\begin{array}{l}
T+\frac{1}{2 N_{0}} \sum_{k=1}^{N_{0}} \lambda_{k} \times B_{4 k}\left(r_{k} \varepsilon\right) \frac{\partial T}{\partial t}=\eta \frac{\partial \varepsilon}{\partial t} \\
\varepsilon(t)=H(0)=\left\{\begin{array}{l}
0 ; t<0 \\
1 ; t \geq 0
\end{array}\right.
\end{array}\right.
$$

or:

$$
T+\frac{1}{2 N_{0}} \sum_{k=1}^{N_{0}} \lambda_{k} \times B_{4 k}\left(r_{k} \varepsilon\right) \frac{\partial T}{\partial t}=\delta(0)
$$

Where $\delta$ denotes the Dirac delta function.

The BPES protocol ensures the validity of the related boundary conditions expressed through biological conditions, regardless main equation features. In fact, thanks to Boubaker polynomials first derivatives properties: 


$$
\left\{\begin{array}{l}
\left.\sum_{q=1}^{N} B_{4 q}(x)\right|_{x=0}=-2 N \neq 0 \\
\left.\sum_{q=1}^{N} B_{4 q}(x)\right|_{x=r_{q}}=0
\end{array}\right.
$$

and:

$$
\begin{gathered}
\left\{\begin{array}{l}
\left.\sum_{q=1}^{N} \frac{d B_{4 q}(x)}{d x}\right|_{x=0}=0 \\
\left.\sum_{q=1}^{N} \frac{d B_{4 q}(x)}{d x}\right|_{x=r_{q}}=\sum_{q=1}^{N} H_{q}
\end{array}\right. \\
\text { with: } H_{n}=B_{4 n}^{\prime}\left(r_{n}\right)=\left(\frac{4 r_{n}\left[2-r_{n}^{2}\right] \times \sum_{q=1}^{n} B_{4 q}^{2}\left(r_{n}\right)}{B_{4(n+1)}\left(r_{n}\right)}+4 r_{n}^{3}\right)
\end{gathered}
$$

boundary conditions are inherently verified.

The BPES solution is obtained through four steps:

$\sqrt{ }$ Determining the set of the pondering real coefficients, $\left.\lambda_{k}\right|_{k=1 . . N_{0}}$ which optimize the fitting of Fig. 2.

$\checkmark$ Integrating, for a given value of $N_{0}$, the whole expressions given by Eq. (12) along time domain.

$\sqrt{ }$ Writing the time dependent solution for stress as a sum of solutions to the $N_{0}$ elementary $k$-indexed equations:

$$
2 N_{0} T+\lambda_{k} \times B_{4 k}\left(r_{k} \varepsilon\right) \frac{\partial T}{\partial t}=2 N_{0} \delta(0)
$$

$\sqrt{ }$ Incrementing $N_{0}$.

$\sqrt{ }$ Testing the convergence of the coefficients $\left.\lambda_{k}\right|_{k=1 . . N_{0}}$.

\subsection{Plots and Discussion}

Plots of the solution are gathered in Fig. 3.

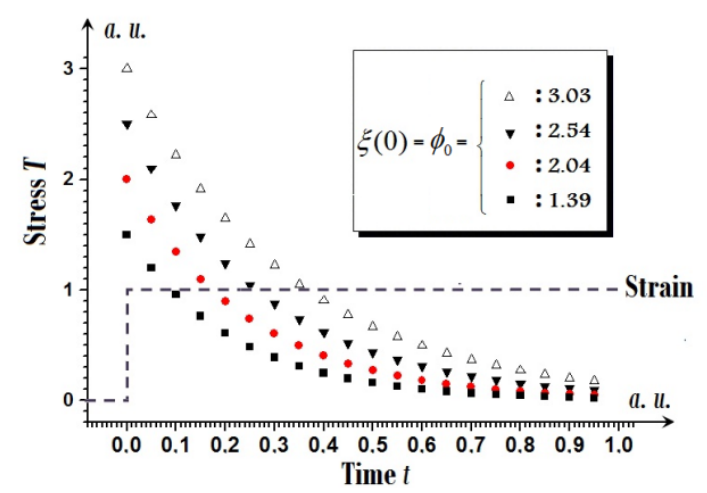

Figure 3. Model stress response to a Heaviside step excitation for different values of $\xi(0)$
The obtained profile (Fig. 3) is in good agreement with the exponential responses recorded by Schmidt et al.[22,23], Oldham et al. [24], Padovan[25], Singh et al.[26] and Yuan et al.[37]. In this solution, and oppositely to some recently performed results, there was no free constant at the level of the initial increment, since all conditions have been fulfilled inherently through the application of the Boubaker Polynomials Expansion Scheme BPES[12-21]. Pipkin et al.[11] discussed a similar solution of the same problem for a Maxwell fluid. The free constant has been determined by means of the boundary fluid layer thickness[11] and the related model required some additional initial conditions apart from the requirement i. e. that the fluid was initially at rest or assuming that the time derivative of velocity is zero at time $t=0$ as proposed by Rajagopal et al.[28-31]. In such models, the unexpected vanishing of the time derivative of the velocity at $t=0$ obviously corresponds to a non-realistic absence of the shear stress. This anomaly has corrected in the actual model through the conditions expressed by Eq. (11-14).

An additional ote concerns the fluid behavior with decreasing values of $\xi(0)$. In fact case when the $\xi(0)$ decreases, tends to zero, the results for Newtonian fluids[32-37] is recovered.

\section{Conclusions}

In this study, the exact and approximate expressions for the response of an enhanced a Maxwell fluid model have been established. As a consequence, terms of shear stress response. In the special case when the retardation time decreases, the results for Newtonian fluids have been recovered. In the proposed model, we had to assume that the stresses in the individual constituents and also the body as a whole are the same. We had also assumed the additive decomposition of the strain of the constituents. Such an assumption is questionable if large strains are involved and we need to consider fluid deformation gradients multiplicative decomposition. This is the object of further studies. the changing of the relaxation time with time is outlined in.

\section{REFERENCES}

[1] J.C. Maxwell, On the dynamical theory of gases, Philosophical Transactions of the Royal Society London A157 (1866) 26-78.

[2] K.R. Rajagopal, A.R. Srinivasa, A thermodynamic framework for rate type fluid models, Journal of Non-Newtonian Fluid Mechanics 88 (2000) 207-227.

[3] D.R. Bland, Theory of Linear Viscoelasticity, Pergammon Press, Oxford, 1960. 
[4] J.D. Ferry, Viscoelastic Properties of Polymers, third ed., John Wiley \& Sons, New York, 1980.

[5] I.J. Rao, K.R. Rajagopal, On a new interpretation of the classical Maxwell model, Mechanics Research Communications 34 (2007) 509-514.

[6] R.G. Larson, S.J.Muller, E.S.G. Shaqfeh, The effect of fluid rheology on the elastic Taylor-Couette instability, J.Non-Newt.Fluid Mech. 51 (1994) 195-225.

[7] R.A. Keiller, Numerical instability of time-dependent flows, J. Non-Newt. Fluid Mech. 43 (1992) 229-246.

[8] V.A. Gorodtsov, A.I. Leonov, On a linear instability of a plane parallel Couette flow of viscoelastic fluid, J. Appl. Math.Mech. 31 (1967) 310-319.

[9] J. Málek, K.R. Rajagopal, A thermodynamic framework for a mixture of two liquids.Nonlinear Anal.-Real World Appl. 9 (2008), 1649-1660.

[10] C.W. Macosko. Rheology: Principles, Measurements, and Applications. Wiley-VCH, NewYork, USA, 1994.

[11] A. C. Pipkin, T. G. Rooers, A nonlinear integral representation for viscoelastic behaviour, J. Mech. Phys. Solids 16 (1968) 59-63

[12] J. Ghanouchi, H. Labiadh and K. Boubaker, An attempt to solve the heat transfert equation in a model of pyrolysis spray using 4q-order m-Boubaker polynomials Int. J. of Heat and Technology, 26 (2008) 49-53

[13] S. Slama, M. Bouhafs and K. B. Ben Mahmoud,A Boubaker Polynomials Solution to Heat Equation for Monitoring A3 Point Evolution During Resistance Spot Welding, International J. of Heat and Techn., 2008, 26(2), 141-146.

[14] T. Ghrib, K. Boubaker and M. Bouhafs, Investigation of thermal diffusivity-microhardness correlation extended to surface-nitrured steel using Boubaker polynomials expansion, Modern Physics Letters B, 22(2008) 2893-2907

[15] S. Fridjine, K.B. Ben Mahmoud, M. Amlouk, M. Bouhafs, A study of sulfur/selenium substitution effects on physical and mechanical properties of vacuum-grown $\mathrm{ZnS}_{1-\mathrm{x}} \mathrm{Se}_{\mathrm{x}}$ compounds using Boubaker polynomials expansion scheme (BPES),J. of Alloys and Comp., 479 (2009) (1-2), 457-461

[16] C. Khélia, K. Boubaker, T. Ben Nasrallah, M. Amlouk, S. Belgacem, Morphological and thermal properties of $\beta-\mathrm{SnS} 2$ sprayed thin films using Boubaker polynomials expansion, Journal of Alloys and Compounds, 477 (2009) (1-2), 461-467

[17] S. Tabatabaei, T. Zhao, , O. Awojoyogbe, F. Moses, Cut-off cooling velocity profiling inside a keyhole model using the Boubaker polynomials expansion scheme, Int.J. Heat Mass Transfer, 45 (2009) 1247-1255.

[18] A. Belhadj, O. Onyango, N. Rozibaeva, Boubaker Polynomials Expansion Scheme-Related Heat Transfer Investigation Inside Keyhole Model , J. Thermophys. Heat Transf., 23 (2009) 639-642.

[19] P. Barry, A. Hennessy, Meixner-Type results for Riordan arrays and associated integer sequences, section 6: The Boubaker polynomials, J. of Integer Seq., 13 (2010) 1-34.

[20] M. Benhaliliba, Benouis, C.E., Boubaker, K., Amlouk M., Amlouk, A., A New Guide To Thermally Optimized Doped
Oxides Monolayer Spray-grown Solar Cells: The Amlouk-boubaker Optothermal Expansivity $\psi$ ab in the book : Solar Cells - New Aspects and Solutions, Edited by: Leonid A. Kosyachenko,[ISBN 978-953-307-761-1], (2011) 27-41.

[21] H. Rahmanov, A Solution to the non Linear Korteweg-De-Vries Equation in the Particular Case Dispersion-Adsorption Problem in Porous Media Using the (BPES), Studies in Nonlinear Sciences, 2 (1) (2011) 46-49.

[22] A.Schmidt, L.Gaul, FE implementation of viscoelastic constitutive stress-strain relations involving fractional time derivatives. In: Constitutive Models for Rubber II. A.A. Balkema Publishers, Tokyo, 2001, 79-89.

[23] A.Schmidt, L.Gaul, On the numerical evaluation of fractional derivatives in multi-degree-of-freedom systems. Signal Processing 86(10), 2006, pp. 2592-2601.

[24] K. B. Oldham, J. Spanier, The Fractional Calculus. Academic Press, New York and London, 1974.

[25] J. Padovan, Computational algorithms for FE formulations involving fractional oper., Comp. Mech.2(1987) 271-287.

[26] S. J. Singh, K. Chatterjee, Galerkin Projections and Finite Elements for Fractional Order Derivatives.Nonlinear Dynamics 45 (1-2) (2006), 183-206.

[27] L. Yuan, O. Agrawal, A Numerical Scheme for Dynamic Systems Containing Fractional Derivatives.Journal of Vibration and Acoustics, 124 (2002) 321-324.

[28] K.R. Rajagopal, On Implicit constitutive Theories, Applications of Mathematics 28 (2003) 279-319.

[29] K.R. Rajagopal, Elasticity of Elasticity, Zeitschrift fur Angewandte Mathematik und Physik (2007).

[30] K.R. Rajagopal, A.R. Srinivasa, On the response of non-dissipative solids, Proceedings of the Royal Society A-Mathematical Phys. and Engin. Sci. 463 (2007) 357-367.

[31] K.R. Rajagopal, On implicit constitutive theories for fluids, Journal of Fluid Mechanics 550 (2006) 243-249.

[32] D. Quemada. Rheological modelling of complex fluids. I. The concept of effective volume fraction revisited. The European Physical J. Applied Physics, 1(1998)119-127.

[33] D. Quemada, Rheological modelling of complex fluids: II. Shear thickening behavior due to shear induced flocculation. The European Physical Journal Applied Physics, 2 (1998) 175-181 doi:10.1051/epjap:1998170.

[34] D. Quemada, Rheological modeling of complex fluids: III. Dilatant behavior of stabilized suspensions. The European Physical J. Applied Physics, 3 (1998), 309-320 D. Quemada, Rheological modelling of complex fluids: IV: Thixotropic and "thixoelastic" behaviour. The European Physical J. Applied Physics, 5 (1999) 191-207.

[35] H. Marzougui, H. Saadouli, H. Khlifi and T. Lili, Numerical simulation of compressible turbulent flow using algebraic Reynolds stress model. The European Physical Journal Applied Physics, 35 , (2006) 69-74

[36] C. Bachelet, Ph. Dantan, P. Flaud Indirect on-line determination of the rheological behavior of a power law fluid based on numerical flow simulations. The European Physical Journal Applied Physics, 25 , (2004), 209-217. 